\title{
The new subject at the Czech Technical University in Prague: laboratories of industrial electronics and sensors
}

\section{Tomas Drabek, Jan Holub, Jan Fischer}

Tomas Drabek, Jan Holub, Jan Fischer, "The new subject at the Czech Technical University in Prague: laboratories of industrial electronics and sensors," Proc. SPIE 11144, Photonics and Education in Measurement Science 2019, 1114415 (17 September 2019); doi: 10.1117/12.2530690

SPIE Event: Joint TC1 - TC2 International Symposium on Photonics and Education in Measurement Science 2019, 2019, Jena, Germany 


\title{
The New Subject at the Czech Technical University in Prague: Laboratories of Industrial Electronics and Sensors
}

\author{
Tomas Drabek*a, Jan Holub ${ }^{\mathrm{a}}$, Jan Fischer ${ }^{\mathrm{a}}$ \\ ${ }^{a}$ Czech Technical University, Faculty of Electrical Engineering, Dept. of Measurement, Technicka \\ 2, 16627 PRAGUE 6, Czechia
}

\begin{abstract}
The subject of the Industrial Electronics and Sensors Laboratory has been designed to be focused on the practical part of the teaching. Here we describe its concept, used equipment and task examples. Experience gathered during subject first run is reported, including final students' feedback.
\end{abstract}

Keywords: Education, Laboratory class, sensors, programming, practical teaching, interactive form

\section{INTRODUCTION}

The subject of Industrial Electronics and Sensors Laboratory [1] has been included in the university curricula based on feedback from industry and the students themselves. The Department of Measurements has reflected the demands of long-term cooperating companies and has created several projects and subjects that have focused on the practical side of teaching.

The following projects are worth mentioning, the Embedded Technology Club (ETC) [2], which is aimed at high school students and undergraduates, where each week two hour lesson is devoted to practical electronics. The content of the subject was conceived mainly on the practical knowledge gained by students by involving a number of typical applications in interesting forms, programming skill development and a final individual project elaboration. Such an object where students use the theoretical knowledge gathered while studying at a university was not available in the university curricula or, similar approaches were very limited at the expense of theoretical knowledge.

\section{TEACHING}

As mentioned in the introduction, the focus of the subject is laboratory work and consequently, regular lectures were not planned. The main goal of the lesson was to make the most of the allocated time to acquire practical knowledge by means of different involvements, programming and solving task included in an individual project.

The objective of the laboratories was to acquaint students "by an interactive form" with basic blocks of the industrial sensor system - starting with the sensor itself, through the signal processing circuits, the analogue to digital signal conversion, up to its microprocessor-based software processing. The results delivery to the parent system and their presentation to the system user in the concept of Internet of Things.

*drabeto3@fel.cvut.cz; phone +420-2-2435-3992; fel.cvut.cz

Photonics and Education in Measurement Science 2019, edited by Maik Rosenberger,

Paul-Gerald Dittrich, Bernhard Zagar, Proc. of SPIE Vol. 11144, 1114415

(C) 2019 SPIE · CCC code: 0277-786X/19/\$21 · doi: 10.1117/12.2530690

Proc. of SPIE Vol. 11144 1114415-1 


\subsection{Instrumentation}

The students acquainted themselves with the basic measurement devices in the field of electronics, namely:

- Handheld digital multimeter,

- Laboratory digital multimeter,

- Digital oscilloscope,

- Platform F0 - LAB,

- $\quad$ LEO Firmware.

The last two items will be discussed further in more details. Platform F0 - LAB is based on STM32F042 microcontroller series. It is a software defined instrument where the microcontroller is built into a solderless contact field - breadboard (Figure 1). Students gained the necessary practical experience while building the circuit. The firmware package contains a pulse generator and either a voltmeter or an oscilloscope (Figure 2).

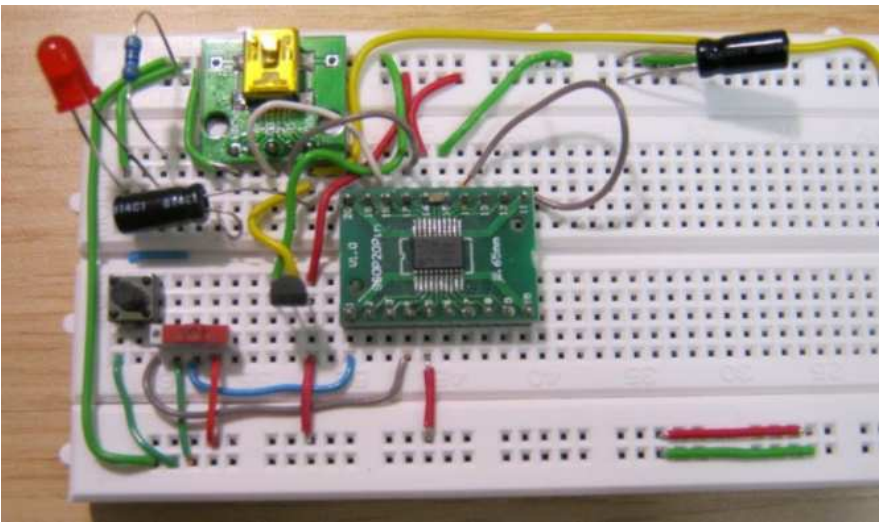

Figure 1. F0 - LAB platform connection in the solderless contact field (breadboard).

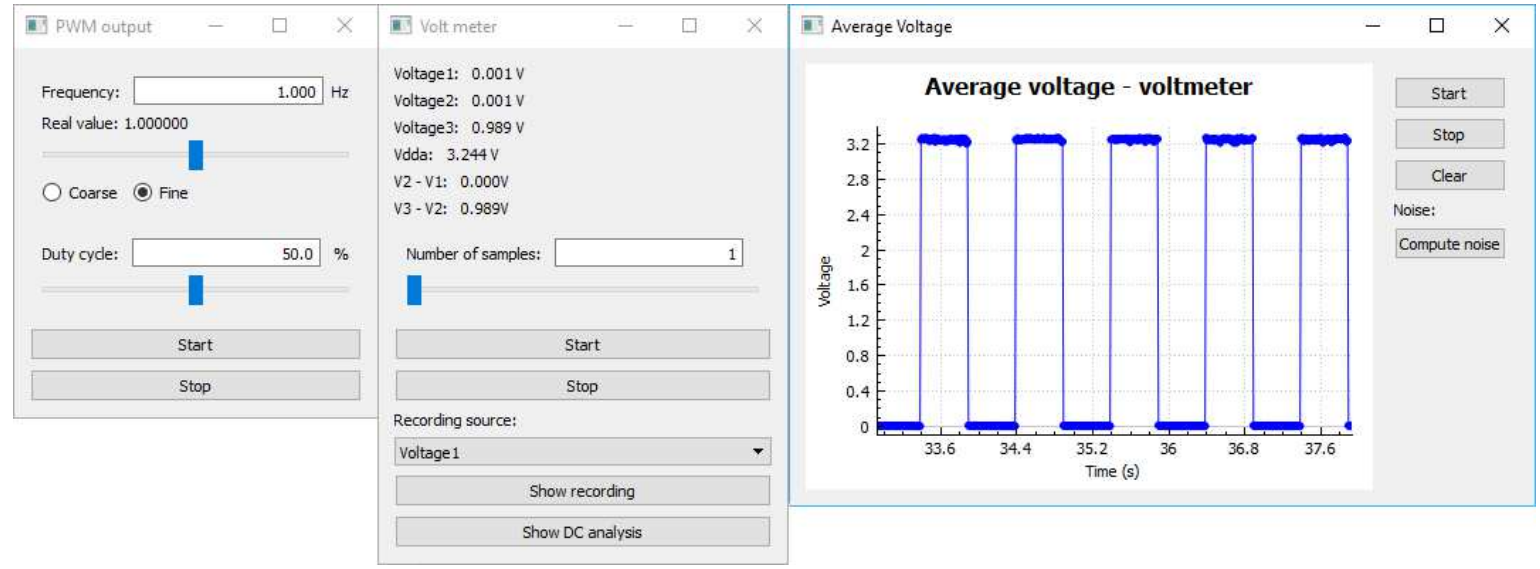

Figure 2. F0 - LAB user interface (PWM output and Voltmeter shown)

Students build a simple, comprehensive and affordable device that replaces expensive devices, and is more than sufficient for required lab work. 
The other device is the LEO (Little Embedded Oscilloscope), which also falls under the category of software defined instruments. For implementation, only the PC and the ST Microelectronics Nucleo STM32F303RE [3] is needed, with the appropriate firmware installed. It includes:

- Two-channel functional generator (provides sinem rectangular, triangular and arbitrary waves),

- Four-channel oscilloscope (sampling frequency 4 Msps, buffer length 50000 bytes),

- Four-channel voltmeter,

- $\quad$ Two adjustable voltage sources $(0-3.3 \mathrm{~V})$.

From the list of functions above, it is obvious that LEO is a more powerful extension of the F0 - LAB. Students used it mainly in the final project, which will be described later.

With the above-mentioned equipment, students are not only acquainted with the devices but are able to actively use it during the laboratories. However, both platforms are fully available to the students during the course and thus the students are able to work on tasks outside the labs.

Both firmware packages have been developed at the Department of Measurement as a school final thesis and serve in several courses taught by the Department both in the Bachelor and Master's study programmes. These firmware packages are also freely available on the department's website [4] accompanied with the wiring instructions. This provides support for the self-employed student work and the development of their knowledge without the need of having basic instruments available.

\subsection{Level of knowledge}

It was soon realized that the entry student's knowledge diverse greatly, therefore, the lab content has been divided into three variants according to the level of experience, so each participant could benefit from the subject, gain new knowledge and skills, and receive a good evaluation.

\subsubsection{Beginner}

Beginner has a zero or only minimal experience with electronics circuitry, single-chip microprocessor programming and electronics in general. It has to be treated with care not to discourage him/her from further interest in practical electronics.

\subsubsection{Advanced}

He or she already attended a similar course before or practices electronic circuit design and development at home, understands basic connections (e.g.: resistor divider, amplifier with operational amplifier, transistor as a switch ...). Advanced student can create and debug simple program for a single chip microcontroller (MBED, Arduino environment, C...).

\subsubsection{Expert}

Expert has previously built more complex structures combining analogue, digital and power electronics (either he has designed them or understands them very well). He or she is capable of programming single-chip microcontrollers (for example C).

\subsection{Laboratory Structure}

The subject was divided into two blocks. The first block was focused on understanding and practice of electronics by means of follow-up tasks and the second block to an individual project, which was adapted to the student knowledge level. 


\subsubsection{Tasks}

Given the diversity of participants' knowledge, it was decided to start from basics to set the same initial knowledge. From using simple components and wirings (LED with resistors, RC circuits...), through a transistor and op-amp circuits, to advanced single-chip microcontroller circuits (using I2C and SPI peripheral buses), connecting simple peripherals to the MCU (LEDs, piezo-transducers, motors), connecting the sensors (optical, magnetic, temperature...) to a PC using the USB-to-Serial port converter (virtual COM-port) and visualizing the measured data. Each assignment was rated, and the students earned the required points for the successful completion of the course, see below. Tasks have been set up to give students a hands-on experience. This approach ensured that the experience gained was gradually used during the entire course and also the final project.

The basic option (Beginner) has been expanded (Advanced) by programming advanced microprocessor peripherals (e.g. graphical display, multiplexed display, sensors with analogue and digital interface, USB interface, Wi-Fi module on solder-less breadboard) and simple circuit board design and assembly (using universal PCBs).

Those students who demonstrated quick and correct task completion were assigned to the Expert level. This group focused on individual projects for most of the semester - working on their own idea or solving a real problem. The project includes a design of a printed circuit board, a circuit board assembly and circuit debugging including programming of a single-chip microcontroller and eventually also PC applications, e.g. for data visualization.

\subsubsection{Project}

The students assigned to the Expert group worked on their project, for approximately the second half of the semester. Typically, they used a Nucleo-board or ARM microcontroller on a solder-free breadboard or universal board, or a proprietary PCB. The project had different topics - for example, sensor data processing (using e.g. Time-of-Flight LIDAR sensors and many others), presentation and transfer of the measured data, or eventually controlling some actuators (e.g. DC motors or stepper motors).

As an example of a simple project, we chose a kitchen timer (Figure 3). It is a self-initiated project where the student designed and programmed the device that served to measure cooking time intervals. The module is controlled by a single button or Wi-Fi module. The measured time spans intervals from $30 \mathrm{~s}$ to $30 \mathrm{~min}$, set in $30 \mathrm{~s}$ intervals. The OLED screen and the Wi-Fi terminal displays the remaining measured time. The end is announced by a simple melody generated by a piezo loudspeaker. The project used F0 - LAB hardware for its implementation and the Wi-Fi module ESP8266 [5] was used to create Wi-Fi.

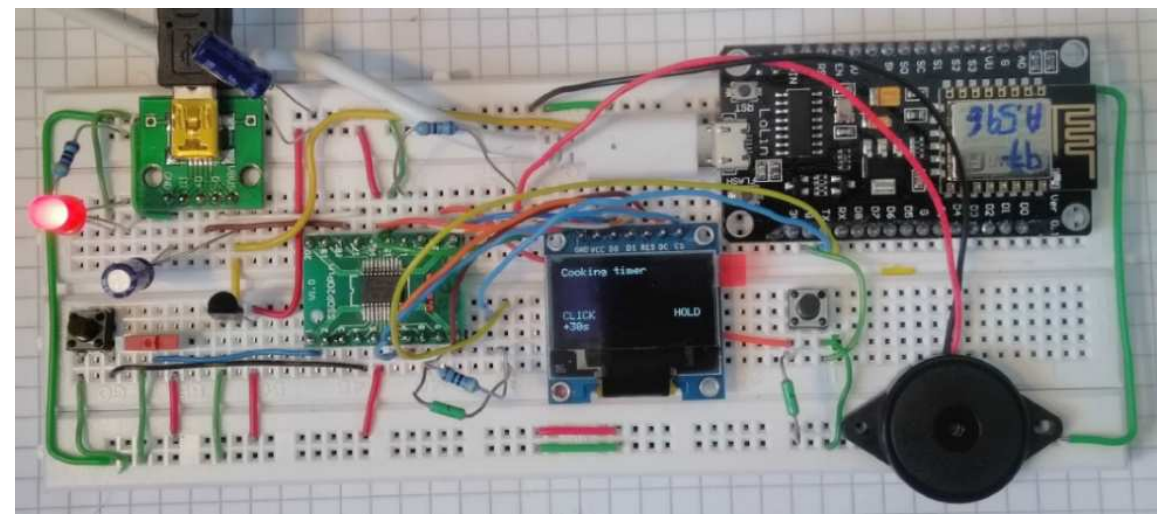

Figure 3. Kitchen timer based on STM32F042 microcontroller

A more complex project was, for example, the conceptual design, assembly and programming of the remotely controlled "miniRover" robot using accelerometers (Figure 4). These are contained in a kit that is available for the students and the robot is remotely controlled by means of simple rf-link. As the hand with the accelerometers attached is tilted, the vehicle receives the data and moves in the desired direction. The bigger the tilt, the faster the robot moves. The project used the F303RE Nucleo board at the rover side but the firmware was completely developed by the student. 


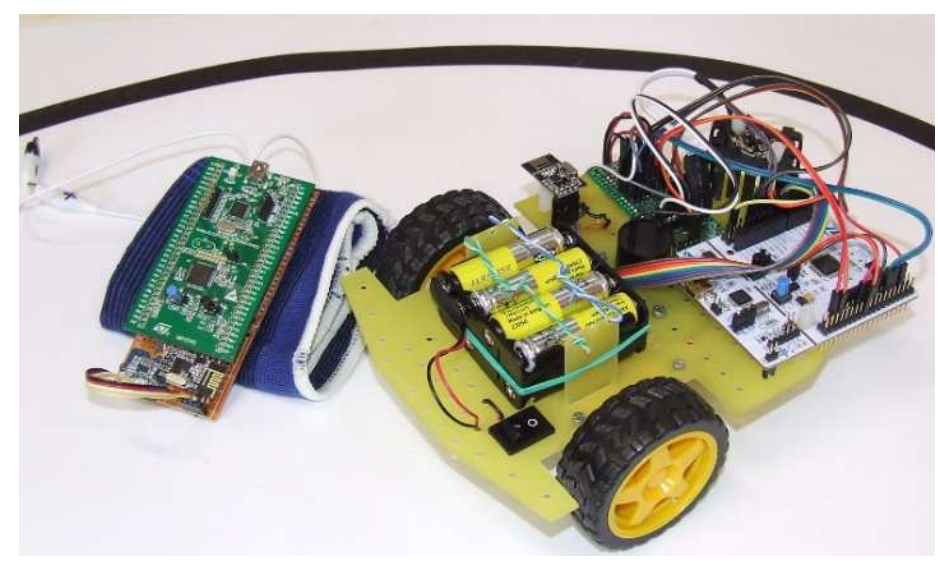

Figure 4. Remorely controlled MiniRover

\section{DISCUSSION}

The subject awards the students with a graded assessment. It is necessary to physically attend the exercises (to obtain points for fulfilling the individual tasks) and put into operation and defend the final project. The number of points from the individual tasks is higher than the minimum score for getting the final A rating. Therefore beginners do not need to be worried about getting worse final mark, and experts do not have to deal with the basics to get enough points. The final presentation of the project is mandatory for all students of the subject, consisting of a submission of the project report (max. 4 pages) and its presentation in maximally 5 minutes.

Even though it is a newly created subject, the participants appreciated it very much, and it was successfully completed by all 37 enrolled students. Perhaps the most valuable feedback comes directly from the students - let us show here the results form the university "Anketa" - an official anonymous student feedback, which is acquired for each subject from all students at the end of every semester:

- All participants rated the subject as "definitely beneficial",

- $\quad 90 \%$ said the difficulty was reasonable.

Let us present two verbal feedbacks that summarizes the subject:

- "Absolutely great subject, for many of us it was the first introduction to electronics and the subject was greatly prepared for it. There were a lot of tasks to choose from, and the more experienced ones found the ones they enjoyed. One of the best subjects for me."

- "I recommend the subject to anyone who has not been yet "picked up" by electronics. Gradually from simpler tasks, we got to the more demanding and overall the subject was very interesting and entertained."

This feedback gives us confidence to further develop this subject.

\section{CONCLUSIONS}

The subject of the Industrial Electronics and Sensors Laboratory was based on feedback from industry and students and focused on the practical part of the teaching. It makes its students acquainted with the basic electrical elements and electronic circuits. During the course, students learned to use common measurement laboratory devices and instruments. They familiarized themselves with the platform F0 - LAB and the LEO firmware, which solved not only common tasks but also their individual project. The course in its first year attended 37 participants, providing very positive final feedback. 


\section{REFERENCES}

[1] <https://www.fel.cvut.cz/en/education/bk/predmety/46/81/p4681706.html> (2. May 2019)

[2] 〈https://embedded.fel.cvut.cz/etc_info $>$ (23. April 2019)

[3] 〈https://www.st.com/content/st_com/en/products/evaluation-tools/product-evaluation-tools/mcu-evaltools/stm32mcu-eval-tools/stm32-mcu-nucleo/nucleo-f303re.html> (2. May 2019)

[4] 〈https://embedded.fel.cvut.cz/kurzy/lpe_sw> (2. May 2019)

[5] 〈https://www.espressif.com/en/products/hardware/esp8266ex/overview> (2. May 2019) 